\title{
Eigenmode analysis
}

\section{for turbomachinery applications}

\author{
Pierre Moinier* Michael B. Giles ${ }^{\dagger}$ \\ Oxford University Computing Laboratory \\ Oxford, United Kingdom
}

September 16, 2005

\begin{abstract}
This paper discusses the numerical computation of unsteady eigenmodes superimposed upon an annular mean flow which is uniform axially and circumferentially, but non-uniform in the radial direction. Both inviscid and viscous flows are considered, and attention is paid to the separation of the eigenmodes into acoustic, entropy and vorticity modes. The numerical computations are validated by comparison to analytic test cases, and results are presented for more realistic engineering applications, showing the utility of the approach for

${ }^{*}$ Research Officer, email: moinier@comlab.ox.ac.uk

†Professor, email: giles@comlab.ox.ac.uk
\end{abstract}


post-processing and for the construction of non-reflecting boundary conditions.

\section{Introduction}

Turbomachinery flows are approximately time-periodic and often conceived as the superposition of an unsteady perturbation on a steady non-uniform mean flow. For aeroelastic analysis, it is important to accurately simulate the features and characteristics of the unsteadiness. To achieve this, two different approaches are typically used. The first is the solution of the unsteady nonlinear equations using standard nonlinear time integration techniques $[2,3,5,11,15]$. The main drawback of these methods is their high computational cost which, in an engineering context, can constitute a major limitation.

The second approach is linear frequency-domain analysis $[8,9,12,14]$, which is computationally much cheaper, partly because it enables the analysis to be performed in a single blade-to-blade passage. Based on the observation that the dominant unsteadiness is small and time-periodic, the unsteady flow field is decomposed into a steady non-uniform mean flow and a sum of time periodic disturbances, each with a distinct known frequency, either a multiple of the blade-passing frequency in forced response, or the blade vibration frequency in flutter analysis. In this approach, non-periodic unsteadiness, such as vortex shedding, is not modelled. It is assumed that this is of relatively 
low amplitude and at a characteristic frequency which is quite distinct from the frequencies of the aeroelastic phenomena being studied. Under these assumptions, the flow equations are linearised in the time domain and transformed into the frequency domain to eliminate the time dependency. It is necessary to first calculate the steady flow about which the linearisation is performed and then perform a separate linear calculation for each frequency of interest.

Although the unsteadiness within a single blade row may be modelled on a domain which extends indefinitely upstream and downstream of the blade row, in practice, numerical solutions must be calculated on a truncated finite domain. It is then important that the unsteadiness radiating away from the blade row must not be artificially reflected at the inlet and outlet boundaries, in order to properly mimic the unbounded character of the far-field. However, spurious reflections can easily be generated at boundaries, if the numerical boundary conditions are inappropriate. To avoid the unsteady flow solution being corrupted by non-physical reflections, Giles [4] introduced non-reflecting boundary conditions for the 2D Euler equations. These were generalised to the 3D Euler equations by Hall et al [10] using a mixed analytical and numerical method to approximate the 3D eigenmodes. In this approach, the unsteady flow is decomposed into upstream and downstream propagating waves, and non-reflecting boundary conditions are enforced by discarding the reflecting components at the far-field boundaries.

The key first step in Hall's work is the computation of the inviscid eigen- 
modes. In related work, Tam and Aurialt [16] have investigated the nature of inviscid eigenmodes in swirling flow, and Cooper and Peake [1] have built on this to perform an asymptotic analysis of the propagation of such modes in ducts with a slowly varying radius.

The present paper addresses the extension of the approach to the 3D Navier-Stokes equations, for which it has proved to be highly effective in reducing the amount of reflection. The details of the non-reflecting boundary conditions and numerical results on engineering applications will be presented in a companion paper [13]. In this paper, the focus is on the computation of the unsteady eigenmodes, and their use for post-processing to analyse and visualise the propagation of acoustic energy within the domain.

Assuming that the mean flow is axially and circumferentially uniform, the numerical method approximates the 3D eigenmodes as the eigenvectors of a General Eigenvalue Problem (GEP) that arises from a discretisation of the linearised Navier-Stokes equations. It will be shown that the direction of propagation of each mode is obtained by looking at the imaginary part of the axial wavenumbers, the eigenvalues of the GEP. The only modes propagating upstream are acoustic eigenmodes, but there are three different families of modes propagating downstream (acoustic, entropy and vorticity modes) and their classification is a delicate matter. Although turbomachinery flows are usually not uniform either in the axial or in the circumferential direction, these assumptions are, in many cases, good enough, particularly at inflow and outflow boundaries. For cases violating these assumptions corrections can be 
applied so that the approach remain valid. Not relevant for the purpose of this paper, they will be introduced in the following paper [13]. The theory will here be applied as such, at different location inside the computational domain and will emphasise the relevancy of the approach, as long as the decomposition is applied away from the blade.

The paper starts with the introduction of the GEP along with the numerical discretisation and followed by the analytical formulations. A method to identify the eigenmodes is then presented and comparisons are shown for inviscid and viscous flows in an annular duct. Finally, applications to realistic geometries exemplify the effectiveness of the approach in post-processing linear unsteady flow fields to reveal information about the propagation and reflection of acoustic energy within turbomachinery simulations.

\section{Inviscid Eigenmode analysis}

We begin with the 3D Euler equations in conservative form and cylindrical coordinates

$$
\frac{\partial \mathbf{Q}_{\mathbf{c}}}{\partial t}+\frac{1}{r} \frac{\partial}{\partial r}\left(r \mathbf{F}_{r}\right)+\frac{1}{r} \frac{\partial \mathbf{F}_{\theta}}{\partial \theta}+\frac{\partial \mathbf{F}_{x}}{\partial x}=\mathbf{G}
$$

$\mathbf{Q}_{\mathbf{c}}$ denotes the conservative variables, $\mathbf{F}_{r}, \mathbf{F}_{\theta}, \mathbf{F}_{x}$ are the fluxes in the radial, circumferential and axial directions, and $\mathbf{G}$ is the source term with Coriolis and centrifugal forces.

Linearising these equations of motion around a mean flow which is axially 
and circumferentially uniform, yields the equation

$$
M \frac{\partial \mathbf{Q}_{p}}{\partial t}+\frac{1}{r} \frac{\partial}{\partial r}\left(r A_{r} \mathbf{Q}_{p}\right)+\frac{1}{r} A_{\theta} \frac{\partial \mathbf{Q}_{p}}{\partial \theta}+A_{x} \frac{\partial \mathbf{Q}_{p}}{\partial x}=S \mathbf{Q}_{p}
$$

where $\mathbf{Q}_{p}$ is the vector of perturbations to the primitive variables (density, velocity and pressure) and $M=\partial \mathbf{Q}_{c} / \partial \mathbf{Q}_{p}$.

Because the matrices $M, A_{r}, A_{\theta}, A_{x}, S$ are all functions of the radius $r$ only, the eigenmodes are of the form

$$
\mathbf{Q}_{p}=\exp (\mathrm{i} \omega t+\mathrm{i} m \theta+\mathrm{i} k x) \mathbf{Q}_{p}(r)
$$

with $\omega$ being a known quantity corresponding to either the frequency of an incoming wave (forced response) or a vibration frequency (flutter). Substituting this into Eq. (2) gives

$$
\mathrm{i} \omega M \mathbf{Q}_{p}+\frac{1}{r} \frac{\partial}{\partial r}\left(r A_{r} \mathbf{Q}_{p}\right)+\frac{1}{r} \mathrm{i} m A_{\theta} \mathbf{Q}_{p}+\mathrm{i} k A_{x} \mathbf{Q}_{p}=S \mathbf{Q}_{p}
$$

Discretising this on a radial grid, with a fourth-difference numerical smoothing, leads to an algebraic equation of the form

$$
\left(\mathrm{i} \omega \widehat{M}+\widehat{A}_{r}+\mathrm{i} m \widehat{A}_{\theta}+\mathrm{i} k \widehat{A}_{x}-\widehat{S}\right) \mathbf{Q}=0
$$

where $\widehat{M}, \widehat{A}_{r}, \widehat{A}_{\theta}, \widehat{A}_{x}$ and $\widehat{S}$ are all matrices of dimension $5 N \times 5 N$ and $\mathrm{Q}$ a vector of length $5 N$, with $N$ being the number of radial grid points. 
On the outer annulus, the radial velocity must be zero, and the same condition applies also on the inner annulus for the case of annular ducts. For cylindrical ducts, the appropriate b.c. at $r=0$ for the unsteady perturbations $\rho, u_{r}, u_{\theta}, u_{x}, p$ depends on the circumferential mode number $m$ :

- $m=0: u_{r}=u_{\theta}=0$,

- $m=1: \rho=u_{x}=p=0$,

- $m>1: \rho=u_{r}=u_{\theta}=u_{x}=p=0$.

These boundary conditions are enforced by removing the corresponding rows and columns of the matrices in Eq. (4) which in turn reduces the size of each vector $\mathbf{Q}$ (e.g. for annular ducts, $\operatorname{dim}(\mathbf{Q})=5 N-2$ ). This now defines an eigenvalue/eigenvector problem solved using routines from the linear algebra library LAPACK ${ }^{1}$ to determine the axial wavenumbers $k$ and the corresponding eigenvectors $\mathbf{Q}$.

\section{Extension to Viscous}

For viscous applications, viscous flux terms must be added to the eigenmode analysis. In addition, the mean flow will exhibit a boundary layer profile that the eigenmodes must take into account if one wishes an accurate modal decomposition of the flow perturbations. Since the mean flow varies only in the radial direction, and assuming that the Reynolds number is very large,

\footnotetext{
${ }^{1}$ www.netlib.org/lapack
} 
as is the case for turbomachinery, it is considered that the gradient of the unsteady flow is predominantly in the radial direction. Consequently, the only significant viscous flux terms are stress terms $\tau_{r r}, \tau_{x r}, \tau_{\theta r}$ and the heat transfer term $q_{r}$, where

$$
\tau_{r r}=\mu \frac{\partial V_{r}}{\partial r}, \quad \tau_{x r}=\mu \frac{\partial V_{x}}{\partial r}, \quad \tau_{\theta r}=\mu r \frac{\partial}{\partial r}\left(\frac{V_{\theta}}{r}\right), \quad q_{r}=-k \frac{\partial T}{\partial r}
$$

$T$ denotes the temperature and is linked to the pressure and the density by the equation of state for a perfect gas. The total viscosity is $\mu=\mu_{l}+\mu_{t}$, where $\mu_{l}$ and $\mu_{t}$ denote the laminar and turbulent viscosity, respectively. The thermal conductivity is given by $k=c_{p}\left(\mu_{l} / P r_{l}+\mu_{t} / P r_{t}\right)$, with $\operatorname{Pr}_{l}$ and $P r_{t}$ being the laminar and turbulent Prandtl numbers.

Discretising these additional viscous flux terms yields the modified GEP,

$$
\left(\mathrm{i} \omega \widehat{M}+\widehat{A}_{r}+\mathrm{i} m \widehat{A}_{\theta}+\mathrm{i} k \widehat{A}_{x}-\widehat{S}-\widehat{V}\right) \mathbf{Q}=0
$$

The wall boundary conditions also have to be modified, imposing no-slip conditions on the velocity. An adiabatic boundary condition is assumed for the heat equation.

\section{Eigenmode identification}

Figure 1 presents the computed wavenumbers $k$ for a test case with uniform inviscid mean flow with Mach number $M=0.5$ in an annular duct with 
inner/outer radius ratio $\lambda=0.5$. The frequency is $\omega=10$, non-dimensionalised by the outer radius and the speed of sound, and the circumferential mode number is $m=1$.

The wavenumbers are each identified as belonging to one of four categories: upstream and downstream propagating acoustic modes, and downstream propagating entropy and vorticity modes. The purpose of this section is to explain how this classification is performed.

Assuming physically stable modes, the upstream propagating acoustic modes are identified in a relatively straight-forward way by looking at the sign of the imaginary part of the wavenumbers. Considering a wave of the form $e^{\mathrm{i} \omega t+\mathrm{i} k x+\mathrm{i} m \theta}$, we can re-write it as $e^{\mathrm{i}\left(\omega t+k_{r} x+m \theta\right)} e^{-k_{i} x}$, where $k_{r}$ and $k_{i}$ are the real and imaginary components of $k$, respectively.

If $k_{i}$ is positive, it corresponds to an evanescent mode with an amplitude which decays exponentially downstream, in the direction of increasing $x$. This can be considered to be a generalised downstream propagating mode, since it corresponds to a limited downstream propagation of a disturbance introduced at an upstream boundary. Similarly, if $k_{i}$ is negative, it corresponds to a upstream propagating evanescent mode.

The difficult case, analytically, is the one in which $k_{i}$ is zero. In this case, one must consider the group velocity, $-\partial \omega / \partial k$. If this is positive, then it is downstream propagating, while if it is negative it is upstream propagating. To avoid the practical difficulty of evaluating the group velocity, we instead introduce a small negative imaginary component to the frequency, 
$\omega_{i}=-10^{-5} \omega_{r}$, corresponding to a very slow exponential growth in time of the eigenmode. This perturbs the wavenumber, giving $k_{i} \approx \frac{\partial \omega}{\partial k} \omega_{i}$. Thus if $k_{i}$ is positive, then it is a downstream propagating mode, while if it is negative it is an upstream propagating mode, exactly the same as for the evanescent modes. Hence, with the introduction of the small negative $\omega_{i}$, the test for the direction of propagation is simply to check the sign of $k_{i}$.

This allows the identification of the upstream propagating acoustic eigenmodes. To distinguish between the three different families of downstream propagating modes, it is necessary to look at the eigenvectors of density $(\rho)$, velocity and pressure $(p)$ perturbations at the different radial nodes. The acoustic modes are identified on the basis that they involve significant pressure perturbations. Accordingly, they are defined to be the modes with the largest values of $\|p\|^{2}$, with the number of modes being equal to the number of upstream propagating modes. In the case of annular ducts with a number of radial grid points equal to $N$, the number of upstream propagating acoustic modes is $N$. The entropy modes are then identified as being the $N$ remaining modes with the largest entropy perturbations $\left\|p-c^{2} \rho\right\|^{2}$, where $c$ is the local speed of sound of the mean flow. The rest of the modes are defined to be vorticity modes, ie $2 N-2$ for an inviscid problem and $2 N-6$ for a viscous problem. The above quantification corresponds to what is expected when the flow is axially subsonic which is normal for turbomachinery problems. For other regimes, the numbers may differ, however, the analysis is expected to work in the same way, since the implementation does not take 
the Mach number into account.

For physically unstable modes, such as vortex sheet instabilities or TollmienSchlichting (TS) waves, the above analysis, based on the imaginary part of $k$, will mis-classify them as upstream propagating modes. Although this might cause problems, such features have not yet been encountered in practice in turbomachinery applications.

Figure 1 shows that the classification works well, but is not perfect. Analytically, the entropy wavenumbers should all have value $-\omega / M[4]$, and the results do show a very tight clustering of these wavenumbers. In the absence of the annular end-walls, the vorticity modes would all have the same wavenumber, but the presence of the walls, and the influence of the numerical smoothing, leads to a spreading out of the values. A couple of the vorticity modes appear to be mis-identified as acoustic modes. These are modes with a very rapid variation in the radial direction, and it is probably an artefact of the numerical smoothing that causes a significant pressure variation in the eigenmodes. There are four "cut-on" acoustic modes with $k_{i}$ almost zero, two corresponding to acoustic modes propagating upstream, and two propagating downstream. The other "cut-off" acoustic modes are evanescent modes. There are two cut-off downstream propagating acoustic modes which have been mis-identified as entropy modes. These are modes with a very large radial variation, leading to significant entropy production due to numerical smoothing.

This method for classifying the eigenmodes seems to work well in real 
turbomachinery applications, such as the ones to be presented later. However, in these applications the theoretical basis for the identification is less solid. If mean flow has a significant swirl component (corresponding to axial vorticity), or radial variation in the axial velocity (corresponding to circumferential vorticity), then the acoustic modes have within them a significant vortical component due to the displacement and stretching of the mean flow vorticity $[1,16]$. Similarly, the vorticity modes have an increasingly large pressure perturbation. Nevertheless, the identification methodology appears adequate for the purposes of both post-processing and the construction of non-reflecting boundary conditions.

\section{Analytic Validation}

\section{Inviscid analysis}

In the case of uniform axial mean flow with Mach number $M$ in an annular duct, linear pressure perturbations satisfy the convected wave equation,

$$
\left(\frac{\partial}{\partial t}+M \frac{\partial}{\partial x}\right)^{2} p=\nabla^{2} p, \quad \lambda<r<1
$$

when non-dimensionalised by the uniform speed of sound and the outer duct radius. The condition of zero radial velocity at the two endwalls leads to the boundary conditions

$$
\frac{\partial p}{\partial r}=0 \text { at } r=\lambda, 1
$$


Looking for eigenmodes of the form

$$
p(x, \theta, r, t)=\exp (\mathrm{i} k x+\mathrm{i} m \theta+\mathrm{i} \omega t) P(r),
$$

leads to the Bessel equation

$$
\frac{1}{r} \frac{\mathrm{d}}{\mathrm{d} r}\left(r \frac{\mathrm{d} P}{\mathrm{~d} r}\right)+\left(\mu^{2}-\frac{m^{2}}{r^{2}}\right) P=0, \quad \lambda<r<1
$$

where

$$
\mu^{2}=(\omega+M k)^{2}-k^{2}
$$

with $\frac{\mathrm{d} P}{\mathrm{~d} r}=0$ on $r=\lambda, 1$. The general solution of the o.d.e. is

$$
P(r)=a J_{m}(\mu r)+b Y_{m}(\mu r)
$$

where $J_{m}$ and $Y_{m}$ are Bessel functions. The b.c.'s give

$$
\left(\begin{array}{cc}
J_{m}^{\prime}(\mu \lambda) & Y_{m}^{\prime}(\mu \lambda) \\
J_{m}^{\prime}(\mu) & Y_{m}^{\prime}(\mu)
\end{array}\right)\left(\begin{array}{l}
a \\
b
\end{array}\right)=0,
$$

so a non-trivial solution requires that the matrix in the above equation has zero determinant. This yields a set of real values for $\mu$ and by solving the quadratic Eq. (6) we obtain $k$.

Figure 2 presents results for the same test case as Fig. 1, showing a comparison between the computed numerical wavenumbers and the analytic 
values for the first 12 acoustic eigenmodes, 6 in each direction.

Despite the limited number of radial points used in the discretisation, the discretisation error is very small for all but the last two modes. Since only the first few modes are wanted for post-processing and the construction of non-reflecting boundary conditions, this is perfectly sufficient. Figure 3 compares the pressure component of the first 3 acoustic eigenmodes propagating upstream. The agreement between the numerical and analytic values is excellent.

\section{Viscous analysis}

In order to validate the viscous eigenmode analysis, approximate analytical solutions of the acoustic eigenmodes for cylindrical ducts have been derived via an asymptotic analysis which is valid at high Reynolds numbers [6]. This asymptotic analysis breaks the domain into three regions:

- a core region in which there is a uniform mean flow, and the unsteadiness is described by potential flow theory;

- a steady boundary layer region in which the mean flow is parallel and non-uniform, and the unsteadiness is described by the linearised inviscid flow equations with very small normal velocity;

- a Stokes sublayer, where the mean flow is approximately stationary, and the unsteadiness is described by the linearised viscous flow equations with a negligible radial pressure variation and normal velocity. 
The effect of both the steady boundary layer and the Stokes sublayer is treated as a small perturbation to the standard eigenvalues and eigenmodes for inviscid potential flow in a duct under the assumption that the nondimensional thickness of the Stokes sublayer (which is proportional to $1 / \sqrt{\omega \mathrm{Re}}$, where $\omega$ is the reduced frequency and Re is the Reynolds number) is much smaller than the steady boundary layer, which in turn is much smaller than unity, which is the non-dimensional radius of the duct.

The viscous test case considers unsteadiness of frequency $\omega=10$ in a cylindrical duct with a core flow of uniform Mach number 0.5. The Reynolds number is chosen to have the extremely high value of $2.5 \times 10^{10}$ to ensure that the Stokes sublayer is much thinner than the steady boundary layer which has thickness 0.002. This clear separation of length scales between the core flow, the steady boundary layer and the Stokes sublayer is important for the asymptotic analysis. Figure 4 shows the steady boundary layer profile next to the outer annulus, and a comparison of the computed and asymptotic pressure component of the first radial eigenmode. Figure 5 shows a comparison of the corresponding computed and asymptotic values for the real and imaginary velocity components, with blow-ups showing the excellent agreement in the steady boundary layer and the Stokes sublayer.

The final validation comes from a comparison of the change in the wavenumber due to the steady boundary layer and viscous effects, taking the difference between the viscous wavenumber value and the corresponding value from inviscid analysis without the steady boundary layer. The computed difference 
is $-0.0364-0.00028 \mathrm{i}$, while the asymptotic value is $-0.0373-0.00031 \mathrm{i}$. It can be proved that the real part is primarily due to the steady boundary layer, while the imaginary part is due to the Stokes sublayer. The agreement is again good, giving confidence in both the numerical evaluation and the asymptotic analysis.

\section{Eigenmode decomposition}

Under the assumption that the mean flow is axisymmetric, a general unsteady flow solution with a given frequency $\omega$ can be represented as a sum of eigenmodes,

$$
Q(x, r, \theta, t)=\sum_{m, n} a_{m n}(x) \exp (\mathrm{i} \omega t+\mathrm{i} m \theta) \mathbf{u}_{m n}(r)
$$

This is a double summation, over the circumferential mode number $m$ and the radial modes $n$ which exist for each value of $m$. The modal amplitude mode $a_{m n}$ will be proportional to $\exp \left(\mathrm{i} k_{m n} x\right)$, where $k_{m n}$ is the axial wavenumber.

Given such a flow solution $Q(x, r, \theta, t)$ computed by a linear frequencydomain analysis as described in the Introduction, the eigenmode decomposition at a particular axial location $x_{0}$ begins by performing a circumferential Fourier transform of the data to obtain the quantities $\widehat{Q}_{m}$ defined by

$$
Q\left(x_{0}, r, \theta, t\right)=\sum_{m} \exp (\mathrm{i} \omega t+\mathrm{i} m \theta) \widehat{Q}_{m}\left(x_{0}, r\right)
$$


From this it follows that

$$
\widehat{Q}_{m}\left(x_{0}, r\right)=\sum_{n} a_{m n}\left(x_{0}\right) \mathbf{u}_{m n}(r) .
$$

If $\widehat{Q}_{m}\left(x_{0}, r\right)$ and the eigenmodes $\mathbf{u}_{m n}(r)$ are sampled at the same set of discrete radial nodes, then the discrete version of this equation is

$$
\widehat{\mathbf{Q}}_{m}\left(x_{0}\right)=\mathbf{A}_{m} \mathbf{a}_{m}\left(x_{0}\right),
$$

where $\mathbf{a}_{m}$ is the vector of modal amplitudes for the different radial modes corresponding to circumferential mode $m$, and $\mathbf{A}_{m}$ is the matrix whose columns are the corresponding discrete eigenvectors. If the number of radial eigenmodes is chosen so that $\mathbf{A}_{m}$ is a square matrix, this can be inverted to obtain

$$
\mathbf{a}_{m}\left(x_{0}\right)=\left(\mathbf{A}_{m}\right)^{-1} \widehat{\mathbf{Q}}_{m}\left(x_{0}\right) .
$$

Repeating this process for each circumferential mode and at differential axial locations enables one to plot the modal amplitudes $a_{m n}(x)$ as a function of axial distance.

The above description is for the post-processing of results obtained from linear frequency-domain solutions. Two such applications are presented in the next section. In the case of nonlinear time-domain calculations, it would be necessary to perform an additional Fourier decomposition in time, to separate out the different frequencies in the solution, and then each of these 
could be treated as described above.

\section{Applications}

The first numerical example concerns a cascade of flat plate stator vanes in an annular duct, as depicted in Fig. 6. The mean flow is inviscid and uniform with the velocity purely in the axial direction. The unsteadiness is due to an analytically defined vortical wave introduced at the inlet of the domain. This problem is known as the category 4 benchmark problem from the $3^{\text {rd }}$ Computational Aeroacoustics Workshop on Benchmark Problems [17]). The results in Fig. 7 show the sound pressure level $\left(\mathrm{SPL}^{2}\right)$ evaluated at the outer wall of the duct, for the first radial acoustic harmonic of a standard linear computation for Fourier modes $-32,-8,16$ and 64 . The incident wave corresponds to circumferential mode 16 . Since there are 24 blades in the cascade, the interaction generates a response solely in modes $m=16+24 p$ for integer $p$.

The harmonic decomposition shows a combination of both propagating and evanescent modes upstream and downstream of the flat plate located at $0<x<0.1$. The modes with approximately constant amplitude are the "cut-on" propagating modes; analytically, their amplitudes should be perfectly constant. The modes with amplitudes which appear to be almost linear in this logarithmic plot are the"cut-off" evanescent modes; the linear

\footnotetext{
${ }^{2} 20$ times the logarithm to the base 10 of the ratio of R.M.S. sound pressure to the reference sound pressure.
} 
logarithmic behaviour corresponds to the expected exponential decay of the modes. The most important point of interest is that the unsteady interaction produces a mode 40 downstream-propagating acoustic wave which is reflected at the downstream boundary into a mode 40 upstream-propagating acoustic wave. There is also a very strong reflection in Fourier mode 16; in this case this is a consequence of the outgoing vortical mode which is not plotted. The reflected mode decays very rapidly away from the boundary and so does not contaminate the computed solution in the neighbourhood of the blade.

The second example concerns the unsteady viscous flow around a turbine outlet guide vane (OGV), shown in Fig. 8, due to an incoming acoustic wave in Fourier mode -10 . Fig. 9a) shows the SPL level of the first radial harmonic for Fourier modes $-28,-10,8$ and 16 when using standard quasi1D non-reflecting boundary conditions. All of the acoustic modes are cuton, upstream and downstream of the blade located in the region $0.64<$ $x<0.84$. Downstream of the blade, there can be seen four acoustic modes propagating downstream, and two propagating back upstream as a result of spurious reflections. These reflections are in the two higher circumferential harmonics for which the quasi-1D non-reflecting boundary conditions are much less effective. Upstream of the blade there are three modes propagating upstream, and two propagating downstream, one of which is the original input disturbance and the other is another spurious reflection. Fig. 9b) shows the great improvement that is achieved through the use of 3D non-reflecting boundary conditions. There is now no spurious reflection at either the inflow 
or the outflow boundaries. The decomposition shown is performed outside the blade location, up to a point, near the blade, where the assumptions are clearly violated. Despite that, the amplitude of the propagation modes remain relatively constant, showing that the error made is quite small.

\section{Conclusions}

In this paper we have described a numerical method for computing unsteady inviscid and viscous eigenmodes in annular ducts with a mean flow which is axially and circumferentially uniform, but can vary radially. The method has been validated by comparison with analytic and asymptotic results for model problems, and then used in two engineering applications as a post-processing tool to display the amplitude of different acoustic modes propagating upstream and downstream of turbomachinery blade rows.

One use of this tool is to identify spurious reflections of outgoing eigenmodes. The extension of this work leads naturally to the implementation of 3D non-reflecting boundary conditions to suppress such reflections. This will be covered in detail in a second paper but one set of results has been included here to show its effectiveness. 


\section{References}

[1] Cooper, A.J., and Peake, N., "Propagation of unsteady disturbances in a slowly varying duct with mean swirling flow", Journal of Fluid Mechanics, 445:207-234, 2001.

[2] Erdos, J.I., Alzner, E., and McNally, W., "Numerical solution of periodic transonic flow through a fan stage", AIAA Journal, 15(11):1559, 1977.

[3] Gerolymos, G.A., "Advances in the numerical integration of the threedimensional Euler equations in vibrating cascades", AIAA Journal of Propulsion and Power, 115(4):356-362, 1993.

[4] Giles, M.B., "Non-reflecting boundary conditions for Euler equation calculations", AIAA Journal, 28(12):2050-2058, 1990.

[5] Giles, M.B., "Stator/rotor interaction in a transonic turbine", AIAA Journal of Propulsion and Power, 6(5):621-627, 1990.

[6] Giles, M.B., and Moinier, P., "Asymptotic analysis of viscous acoustic eigenmodes", In preparation, 2004.

[7] Hall, K.C., "Deforming grid variational principle for unsteady small disturbance flows in cascades", AIAA Journal, 31(5):891-900, 1993.

[8] Hall, K.C., and Crawley, E.F., "Calculation of unsteady flows in turbomachinery using the linearized Euler equations", AIAA Journal, 27(6):777-787, Jun 1989. 
[9] Hall, K.C., and Lorence, C.B., "Calculation of three-dimensional unsteady flows in turbomachinery using the linearized harmonic Euler equations", Journal of Turbomachinery, 115:800-809, October 1993.

[10] Hall, K.C., Lorence, C.B., and Clark, W.S., "Non-reflecting boundary conditions for linearized unsteady aerodynamic calculations", AIAA Paper 93-0882, 1993.

[11] He, L., and Denton, J.D., "3-dimensional time-marching inviscid and viscous solutions for unsteady flows around vibrating blades", Journal of Turbomachinery, 116(3):469-476, 1994.

[12] Lindquist, D.R., and Giles, M.B., "Validity of linearized unsteady Euler equations with shock capturing", AIAA Journal, 32(1):46, 1994.

[13] Moinier, P. and Giles, M.B., "Non-reflecting boundary conditions for 3D viscous flows in turbomachinery", In preparation, 2004.

[14] Ni, R.H., and Sisto, F., "Numerical computation of nonstationary aerodynamics of flat plate cascades in compressible flow", Journal of Engineering for Power, 98:165-170, 1976.

[15] Rai, M.M., "Navier-Stokes simulations of rotor-stator interaction using patched and overlaid grids", AIAA Journal of Propulsion and Power, 3(5):387-396, 1987.

[16] Tam, C.K., and Auriault, L., "The wave modes in ducted swirling flows with mean swirling flow", Journal of Fluid Mechanics, 371:1-20, 1998. 
[17] Third computational aeroacoustics workshop on benchmark problems. Ohio Aerospace Institute,Brook Park/Cleveland,Ohio, November 1999. 

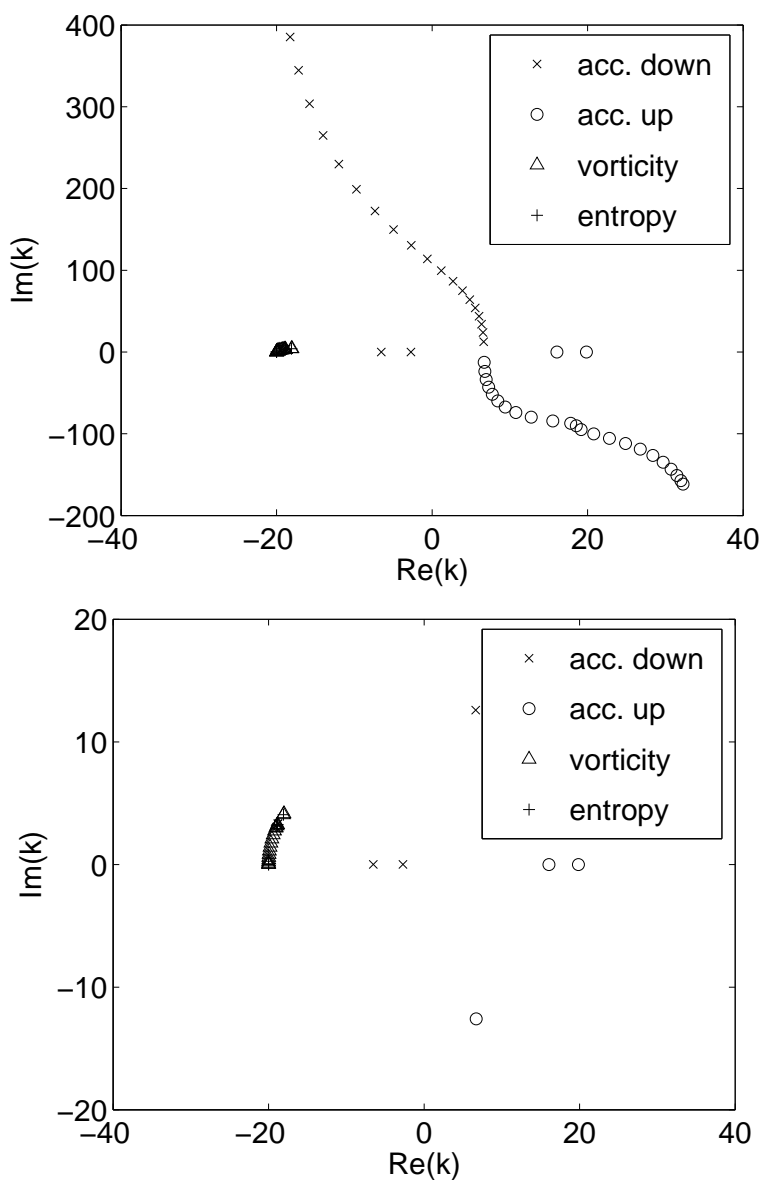

Figure 1: Numerical wavenumbers for inviscid annular duct flow; $r_{\text {inner }} / r_{\text {outer }}=0.5, M=0.5, \omega=10, m=1$. 


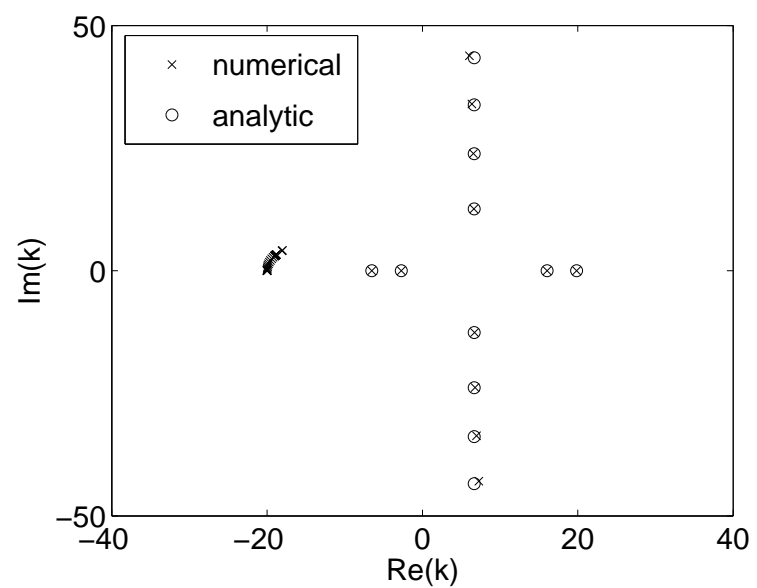

Figure 2: Comparison of numerical and analytic wavenumbers for inviscid annular duct flow; $r_{\text {inner }} / r_{\text {outer }}=0.5, M=0.5, \omega=10, m=1$.
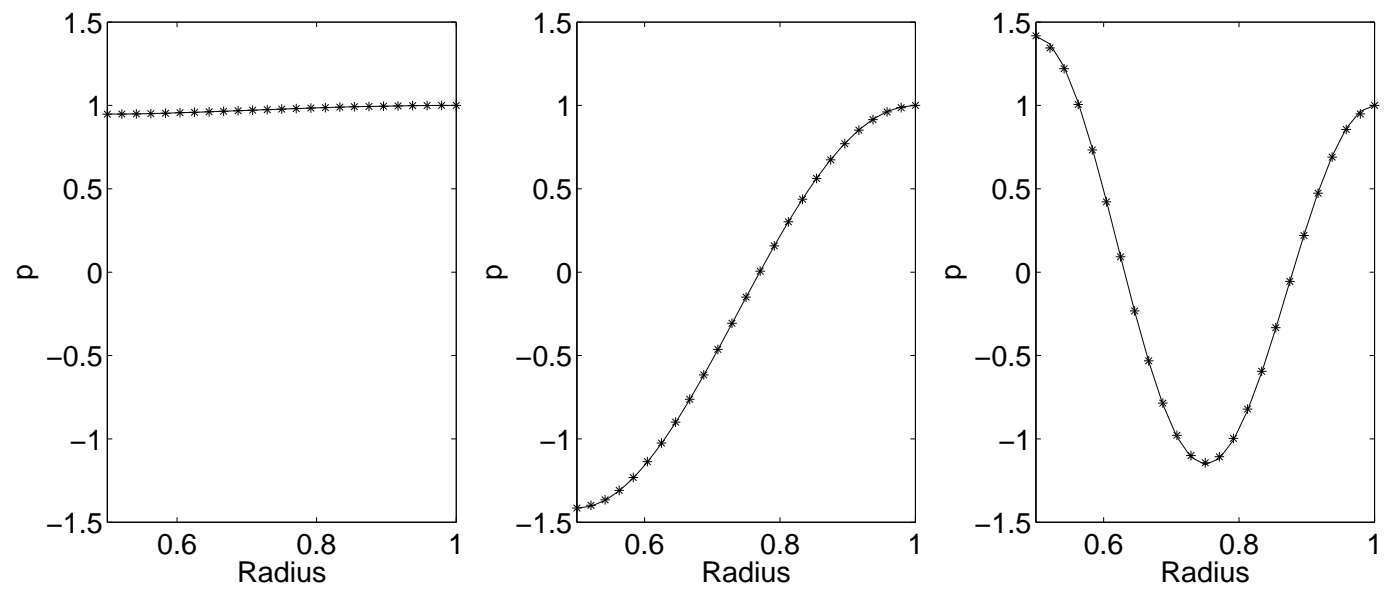

Figure 3: Comparison of numerical (symbol) and analytic (line) pressure eigenmodes for first three upstream-travelling acoustic modes. 

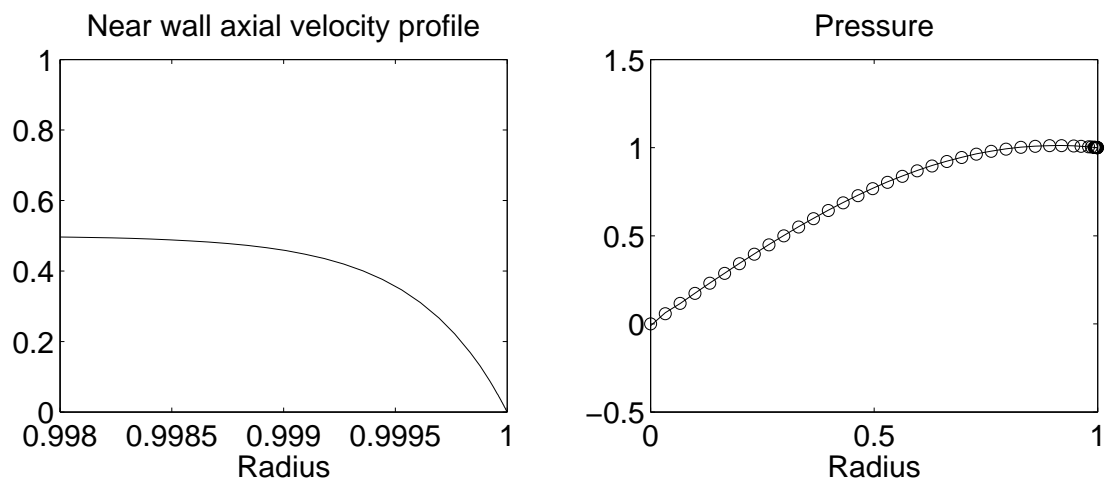

Figure 4: Steady boundary layer velocity profile, and comparison of numerical (symbol) and asymptotic (line) pressure eigenmode for viscous test case. 

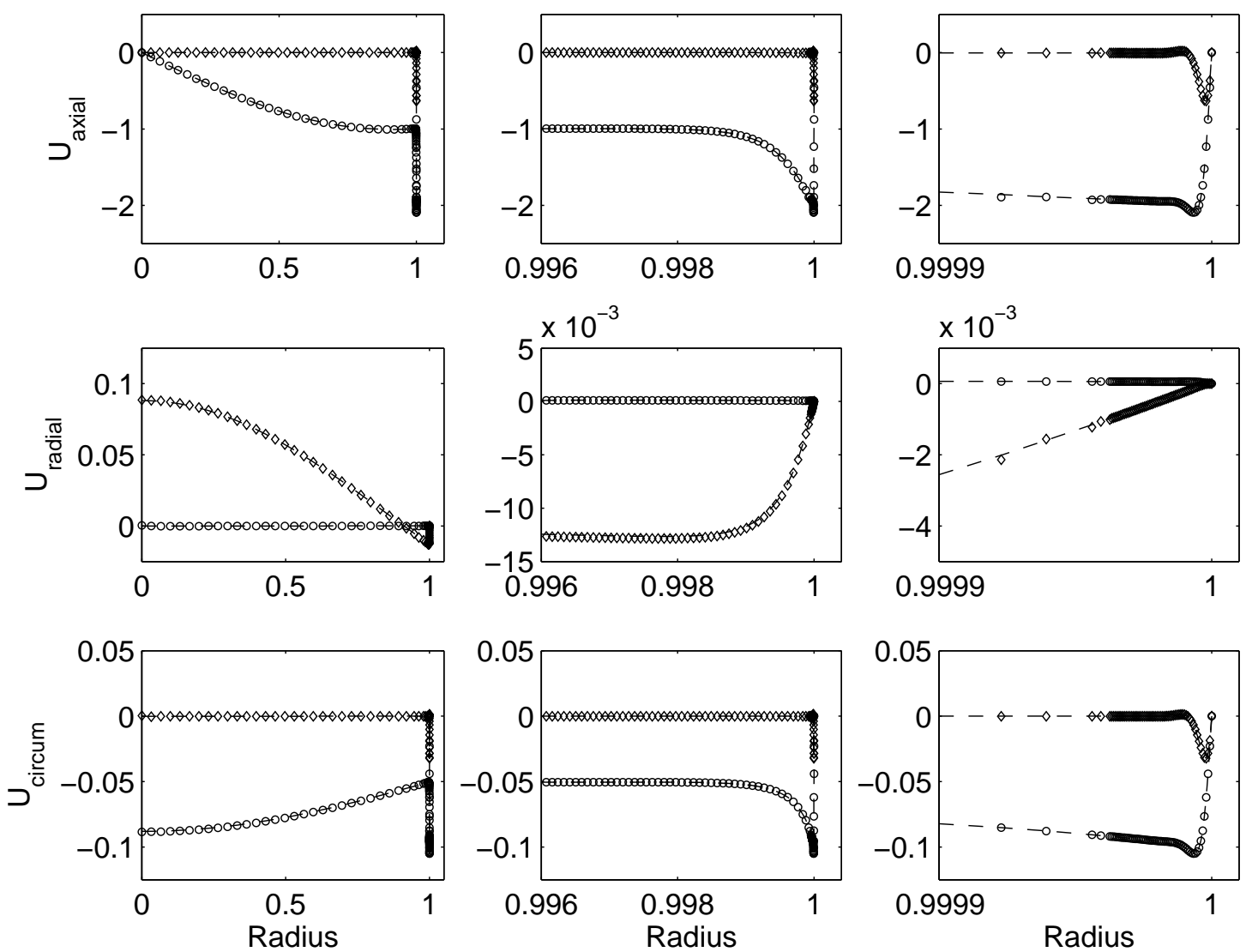

Figure 5: Comparison of the real $(\circ)$ and imaginary $(\diamond)$ numerical and asymptotic (line) values for axial, radial and circumferential velocity components in the core, steady boundary and Stokes sublayer. 


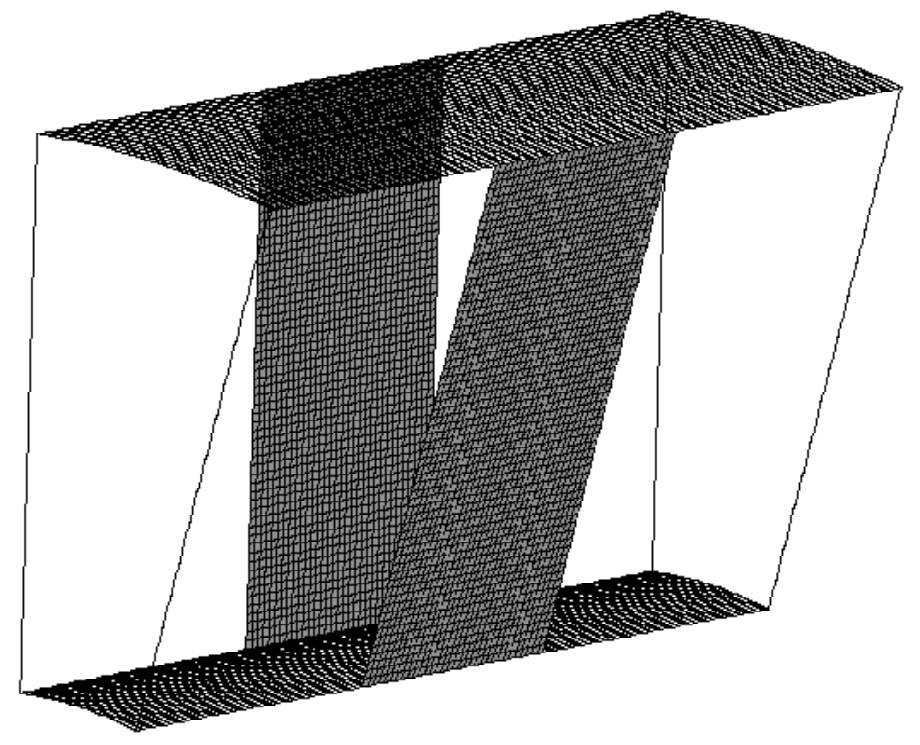

Figure 6: Inviscid annular cascade: geometry and surface grid. 


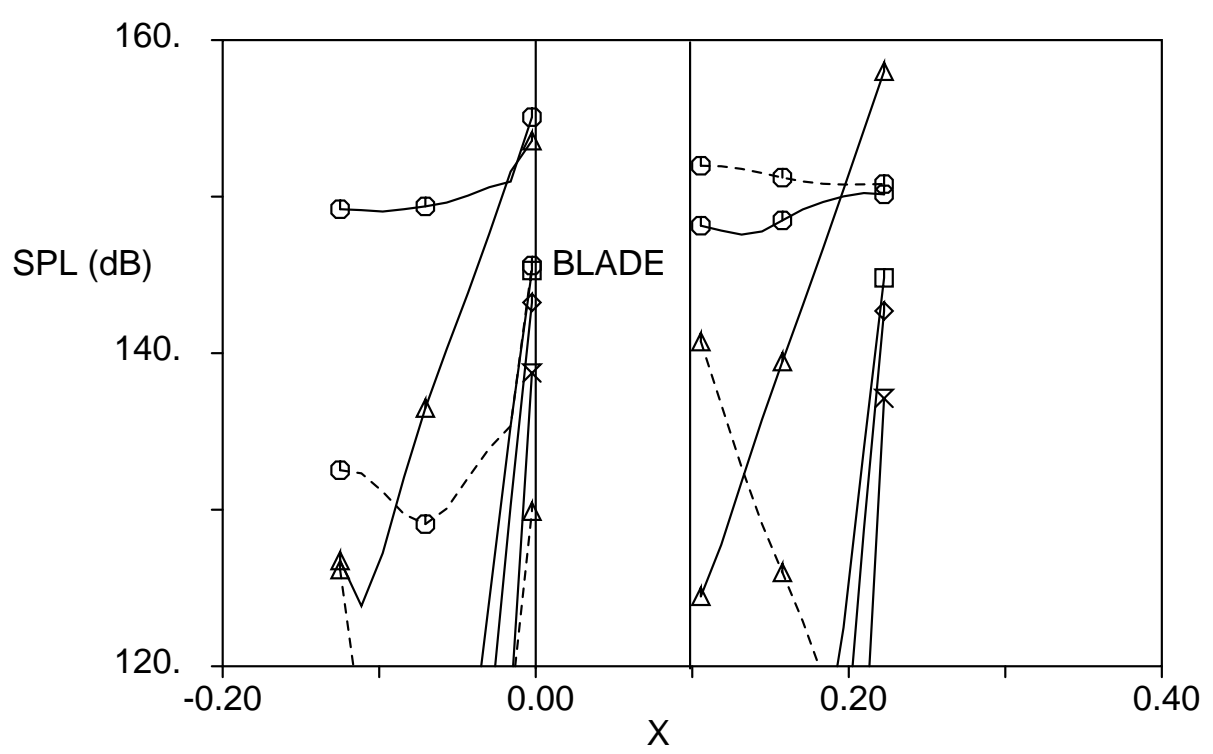

Figure 7: Inviscid annular cascade: amplitude of the first radial harmonic acoustic mode propagating upstream (straight line) and downstream (dashed line) for circumferential Fourier modes $-32(\diamond),-8(\times), 16(\triangle), 40(\circ)$ and $64(\square)$. 


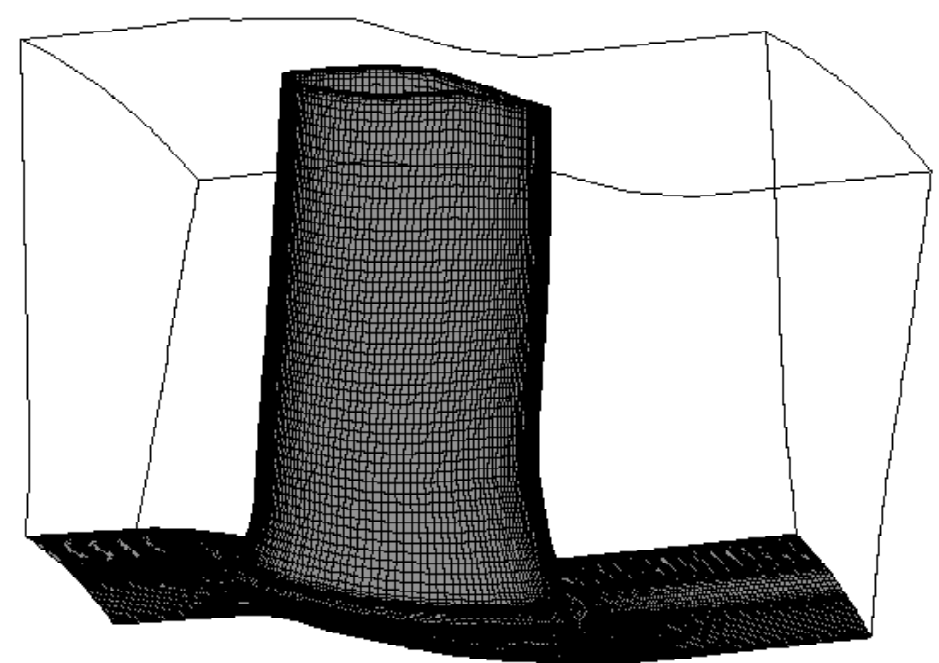

Figure 8: Viscous turbine OGV: geometry and surface grid. 


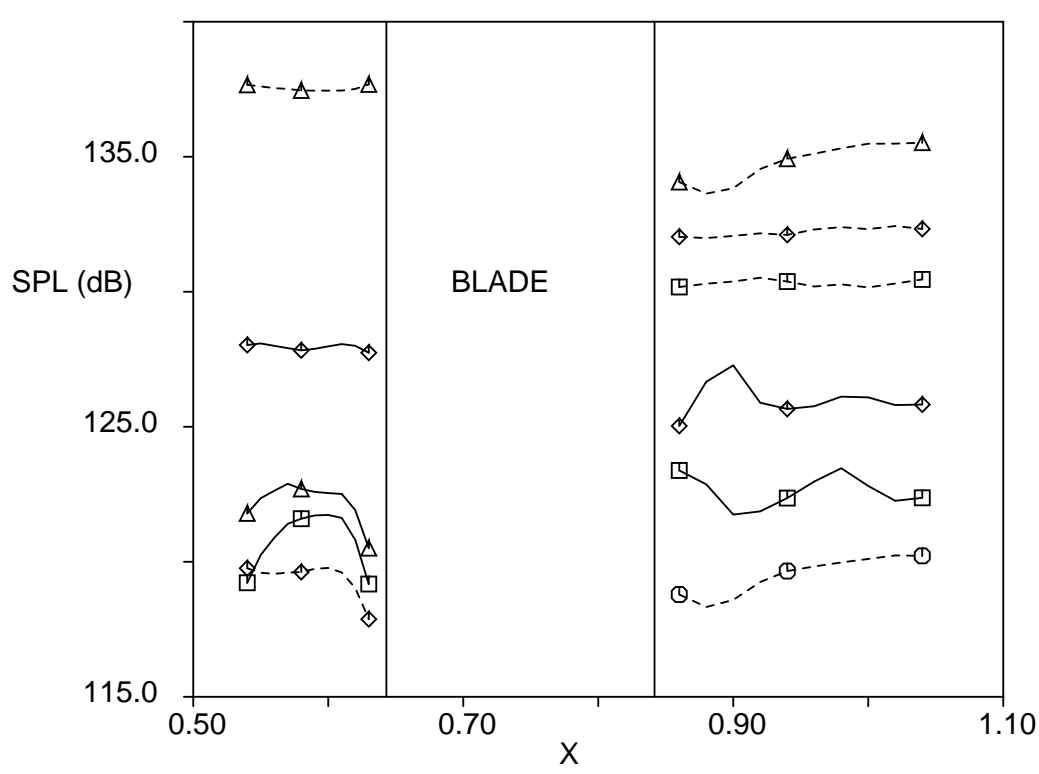

a) quasi-1D non-reflecting b.c.'s

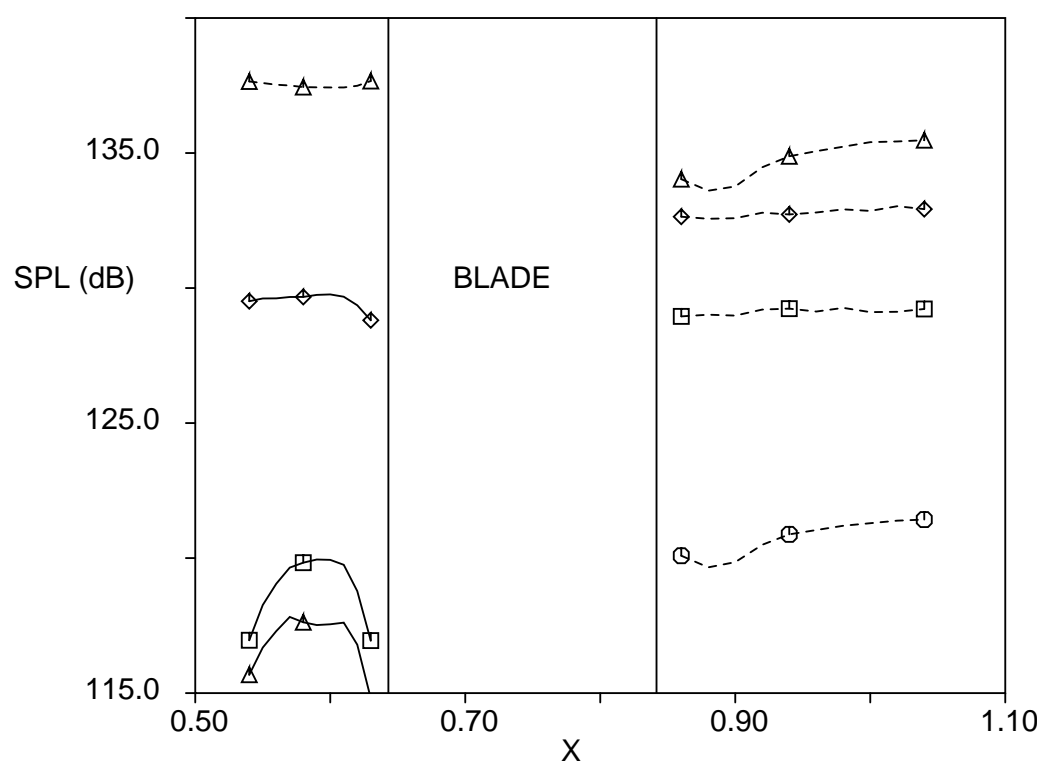

b) 3D non-reflecting b.c.'s

Figure 9: Viscous turbine OGV: amplitude of the first radial harmonic acoustic mode propagating upstream (straight line) and downstream (dashed line) for circumferential Fourier modes $-28(\diamond),-10(\triangle), 8(\circ)$ and 26( $\square)$. 